\title{
L'éducation complexe sous le prisme de la philosophie du care
}

The complex education from philosophy of care approach

\section{Alicia Garcia}

\section{OpenEdition}

\section{Journals}

Édition électronique

URL : http://journals.openedition.org/trema/6318

DOI : $10.4000 /$ trema. 6318

ISSN : 2107-0997

Éditeur

Faculté d'Éducation de l'université de Montpellier

Référence électronique

Alicia Garcia, «L'éducation complexe sous le prisme de la philosophie du care », Tréma [En ligne], 54 I

2020, mis en ligne le 01 décembre 2020, consulté le 14 décembre 2020. URL : http://

journals.openedition.org/trema/6318; DOI : https://doi.org/10.4000/trema.6318

Ce document a été généré automatiquement le 14 décembre 2020.

Trema 


\title{
L'éducation complexe sous le prisme de la philosophie du care
}

\author{
The complex education from philosophy of care approach
}

\author{
Alicia Garcia
}

\section{Introduction}

1 Un très grand nombre de recherches ont déjà éclairé les sources du paradigme éducatif complexe dont Edgar Morin parle, qu'il s'agisse de souligner la façon dont il s'inscrit dans le sillage de la théorie de la pensée complexe ou de s'interroger sur leurs apports à l'éducation du futur. Pourtant, la convergence entre la pensée complexe de Morin et les approches pragmatistes de l'éducation n'a pas été encore abordée. L'objet de cet article porte sur les points de rencontre entre l'éducation complexe et l'éducation basée sur une approche pragmatiste, notamment celle du care.

2 Il est nécessaire de souligner que la convergence entre la pensée pragmatiste et les approches du care a été peu abordée dans le contexte francophone. Alice Le Goff (2013) a traité la question de la relation entre le pragmatisme et le care en soulignant l'importance de la promotion d'expériences participatives comme moyen, à caractère démocratique, pour le développement individuel des élèves. C'est au prisme de l'action que son travail met en lumière les points de rencontre entre le pragmatisme et l'éthique du care. De leur côté, les espagnols Alicia Garcia et Gonzalo Jover (2019) ont également étudié cette question, mais en mettant l'accent sur le rôle des sentiments, notamment le sentiment de bonheur, et l'importance de la perception des élèves sur la finalité de l'éducation ; soulignant l'importance pour les élèves de se sentir "soignés " ('care receiving') pour l'accomplissement d'une éducation plurielle et démocratique.

3 Néanmoins, il est possible d'établir une convergence entre le pragmatisme et la philosophie du care encore plus intime, ressortant dès lors qu'on prend en considération les axes structurants d'une communauté pédagogique pragmatiste dont Ella Flagg Young et John Dewey sont fondateurs (Haddock Seigfried, 1996). Basé sur une recherche en philosophie de l'éducation, cet article vise donc à aborder la question de 
la « relation» dans le "paradigme éducatif complexe». Pour cela, les questions que nous poserons seront les suivantes: Quels sont les principes éthiques, épistémologiques et politiques qui se réfèrent à l'éducation complexe? Quel rôle y joue la relation pédagogique? Peut-on mobiliser une épistémologie, voire une logique de relation basée sur la problématisation capable de répondre d'une façon globale aux besoins de l'enfant, de l'élève et de l'écolier? En s'appuyant sur les travaux de Noddings (2005) et de Tronto (2009), on examine la question de la « relation » sous l'angle de l'éthique du care réinterrogeant et refondant la question « Quoi enseigner? » au cœur de la relation pédagogique.

4 Le concept d'inclusion est extrait du rapport de l'Unesco 2006 : Principes directeurs pour l'inclusion. Il découle de cette conception que les chances de progrès sont plus importantes si on reconnait que les besoins de l'éducation proviennent de la diversité des besoins de tous les élèves et de l'amélioration des résultats éducatifs. Il est mis en évidence qu'une participation dans les processus d'apprentissage, le débat, les attitudes positives est à promouvoir afin de répondre de manière satisfaisante aux défis de l'éducation et des systèmes éducatifs dans un sens intégral en considérant les particularités des élèves, les contextes et les processus non comme des problèmes à résoudre, mais comme des possibilités d'enrichissement de l'apprentissage.

\section{Méthodologie}

5 Pour comprendre le contenu de l'éducation complexe de Morin dans ce qu'elle a de pragmatiste, il faut prendre acte du déplacement épistémologique auquel elle nous convie et du rapport qu'elle nous invite à nouer avec une conception démocratique et inclusive de l'éducation. La méthode mobilisée dans cette réflexion fait appel au structuralisme épistémologique dans un sens encore peu exploité. Inspiré essentiellement des phases qui organisent toute relation du care, ainsi que les dimensions morales qui suivent, selon celles formulées par Joan Tronto dans son ouvrage Un monde vulnérable (Tronto, 2009 : 147-150). Le projet d'éducation complexe de Morin est inséré dans une analyse épistémologique en tant que manifestation d'une relation essentiellement éthique.

6 L'objectif ici n'a pas été de faire une étude comparative entre la pensée complexe d'Edgar Morin et les éthiques du care, ce qui aurait posé des difficultés catégoriales. Pour ouvrir la réflexion, nous avons souhaité mettre en évidence les idées fortes qui structurent le projet d'une éducation complexe et ce qu'elles partagent avec le projet pragmatiste d'une éducation basée sur le care. Or ce que nous analyserons ici est la relation éthique-épistémologique sur laquelle Morin fait pivoter son idée d'éducation et ce qu'il partage avec les philosophies pragmatistes du care. Il s'agit par-là de mettre en lumière les raisons pour lesquelles la complexité du projet de Morin et son apport à la réflexion pédagogique sur le care sont pensés. Tout en soulignant la contribution de ce projet à la promotion de politiques et de pratiques éducatives inclusives.

7 À ce propos, dans un premier temps, nous abordons l'interprétation que fait Morin de la crise de l'éducation. Ensuite, nous procédons à l'analyse de l'appropriation de la théorie générale de systèmes qui va lui permettre de penser l'éducation de façon complexe et de surpasser les fractures qui suivent à la crise de l'éducation ainsi que développer une épistémologie alternative pour, finalement, mettre en relief l'impact que cette épistémologie a pour la création et l'établissement d'une communauté 
éducative inclusive. Le propos est de montrer la forme où l'éducation, dans un sens large et en tant que phénomène humain et social, est prise par Morin comme un système complexe favorisant l'établissement d'une communauté éducative inclusive; l'accent étant mis moins sur les éléments fondamentaux de ce système que sur les relations qui les unissent.

\section{Résultats}

8 C'est au prisme de la pensée complexe d'Edgar Morin que ce texte met en lumière les points de rencontre entre pragmatisme et philosophie du care. Morin élabore une épistémologie éducative dont les approches du care peuvent se réclamer. Cet article explore la façon dont cette épistémologie met en évidence la relation en éducation, conçue comme une organisation «écologique » entre défense des valeurs éthiques du care et promotion du continuum théorie-pratique pédagogique. L'étude des rapports entre la réflexion éducative d'Edgard Morin et l'approche pragmatiste de l'éducation sous le prisme de la philosophie du care permet de mettre en relief l'affinité qui semble bien exister entre la défense des pratiques et des valeurs du care et la valorisation des formes "éco-organisatives» de la pensée complexe permettant un regard et une relation avec l'autre capable de répondre aux défis du contexte de globalisation et d'interconnexion où l'éducation inclusive du futur doit se construire.

9 Le défi de penser l'éducation comme un phénomène complexe pose le problème de l'élargissement des représentations habituelles de «l'acte éducatif» et oblige à revenir à des questions fondamentales comme celle qui nous interroge sur le but de l'éducation et des systèmes éducatifs. Le prisme de l'éducation complexe met en relief que l'éducation englobe d'autres processus que l'instruction aussi importants pour le développement humain. L'analyse du projet d'éducation complexe d' Edgar Morin sous le prisme de la philosophique du care, notamment les réflexions de Joan Tronto et Nel Noddings, permet de montrer la place que l'éducation complexe accorde aux acteurs et leurs expériences et sa valeur pour une éducation dite inclusive. Face aux théories sociologiques de l'éducation « classiques », qui instaurent une coupure épistémologique entre sujets et objets de la connaissance, entre description et évaluation, l'approche philosophique du care, éminemment pluraliste (indissociablement descriptive et évaluative), est capable d'orienter la description de la complexité, qui n'est que l'articulation des différentes relations en éducation (soit au niveau épistémologique soit au niveau pédagogique), par une question qui problématise ce qui vaut pour description factuelle et objective du monde éducatif: qui s'occupe de quoi et comment? À partir de cette question peut être décrite, évaluée et critiquée la dimension aussi bien épistémologique qu'éthique du phénomène éducatif.

\section{Le but de l'éducation complexe face à la crise de l'éducation}

Edgar Morin arrive à la conclusion que la base de la crise que traverse l'humanité est une crise cognitive : notre manière de savoir n'a pas suffisamment développé l'aptitude à contextualiser l'information et à l'intégrer dans un ensemble qui lui donne un sens (Morin 2011: 142). Pour Morin, comme pour d'autres auteurs ${ }^{1}$, l'éducation est en crise. Pour lui l'origine de cette crise consiste à la dissociation entre la culture scientifique et 
la culture humaniste qui a commencé au cours du XVIIe siècle avec la promotion de la méthode rationnelle-scientifique et critique du Discours de la méthode de Descartes et qui a débouché dans une civilisation des dualismes: entre moyens et finalités, entre méthode et connaissance; entre apprendre et transmettre (Molinaro, 2017). Dans Enseigner à vivre (2014), Morin explique la crise de l'éducation comme une crise de civilisation. Le mode compartimenté et fragmenté dans lequel vivent les spécialistes, techniciens, experts, les administrations et les bureaux, conduit inexorablement à la perte de vision d'ensemble, dans laquelle nous travaillons, ainsi que sur la culture dans laquelle nous vivons, ce qui nous fait perdre le sens de la « responsabilité ».

11 Ce qui constitue la fin même de l'éducation pour Morin, du secondaire à l'université est d'« enseigner à vivre ", à vivre sa vie et à vivre ensemble. C'est un nouvel humanisme qui est proposé ici, fondé sur des nouveaux outils de réflexion et d'apprentissages de l'humain. Pour Morin, il est donc nécessaire d'introduire la crise de l'éducation dans un contexte de crise plus vaste qui fait appel à cette appartenance à l'espèce humaine. Or la crise de civilisation dépend des autres crises (culturelle, sociale, sociétale, démocratique, économique) qui dépendent elles-mêmes à leurs tours de la crise de l'éducation. Cette crise de l'éducation et de la civilisation dépendent l'une et l'autre d'une crise de la connaissance qui dépend à son tour des unes et des autres. Morin nous explique cette crise en faisant appel à son étymologie ; le mot crise (en grec "krisis", terme de la médecine hippocratique, qui désignait le moment où une maladie révélait ses symptômes propres, ce qui permettait le juste diagnostic et le remède approprié) renvoie toujours à un système et à son organisation. Tous ces éléments (espèce humaine, civilisation, culture, connaissance donc constituent une poly-crise de multiples visages. Néanmoins, tous ceux-ci sont aussi d'une certaine façon solidaires dans la mesure où ils appartiennent à un même système et ils partagent la même essence vitale, la relation humaine : « la crise de l'éducation doit donc être conçue dans sa complexité propre laquelle renvoie à la crise de la complexité sociale et humaine » (Morin, 2014 : 49).

12 Étendue à l'éducation, la pensée complexe suppose «de réformer la pensée pour réformer l'enseignement et de réformer l'enseignement pour réformer la pensée » (Morin, 1999). Edgar Morin prône ainsi une réforme profonde de l'éducation en général, centrée sur sa mission essentielle telle que l'envisageait Rousseau: "enseigner à vivre». Il s'agit alors pour Morin de permettre à chaque individu d'acquérir une autonomie, de se prémunir contre l'erreur et l'illusion, de pratiquer la compréhension d'autrui, de même que d'affronter les problèmes du "vivre » et les incertitudes de toute vie. Cette éducation, fondamentalement humaniste, doit permettre à chacun de concevoir et de traiter les problèmes fondamentaux et les problèmes globaux. Lorsque Morin s'est mis en devoir de penser l'éducation du futur, une éducation dans laquelle la tâche la plus importante est celle d'articuler, organiser et "percevoir et concevoir le Contexte, le Global (la relation tout/parties), le Multidimensionnel, le Complexe » (Morin, 2008), on prend conscience de l'importance des principes éthiques qui doivent guider l'éducation et sans lesquels elle reste vide de sens (Kant, 1986).

D'après la perspective de l'éducation complexe et du care, la connaissance qui véhicule une éducation qui n'est pas focalisée sur son but, enseigner à vivre, devient une marchandise et atteint l'intégrité morale des populations. La connaissance, quand elle est séparée du vivant et de son cadre social, perd toute sa complexité et n'est qu'une 
connaissance morte, elle devient un objet mercantilisé et échangeable. Une éducation contraire à son objectif "enseigner à vivre" pourra donc être nommée "necroéducation $»^{2}$. La gestion de l'éducation basée sur cette idée, mis en évidence notamment par la transformation et la fragmentation des connaissances et les évaluations standardisées, entraine une nouvelle façon de concevoir l'éducation où la vie humaine perd toute sa complexité. Ainsi conçue, l'éducation devient une connaissance échangeable pour un pouvoir qui fragmente et ne permet pas la communication entre les savoirs et en conséquence ne favorise pas l'ouverture d'esprit en conduisant aussi bien à l'incompréhension du phénomène éducatif dans son ensemble qu'au manque de solidarité entre les différents agents impliqués.

14 La crise de l'éducation peut être donc décrite pour Morin comme le résultat de la destruction de la « solidarité » et de la « responsabilité ». Dans le cas de l'éducation, les enseignants ne connaissent la culture juvénile avec laquelle ils travaillent, comme également les élèves ignorent les problèmes profonds qui affectent le monde enseignant. En même temps, l'éducation manque de réflexivité sur son futur. Or, les requis, sous le prisme de la pensée complexe, ne peuvent être que la compréhension de l'ensemble éducatif, par définition essentiellement complexe.

\section{En quoi l'éducation complexe est pragmatiste?}

Un lecteur découvrant les textes de Morin ne pourra qu'être frappé par la prégnance du vocabulaire biologiciste typique de la Théorie General des Systèmes (TGS) (Bertalanffy, 2012) qu'il n'applique pas seulement aux champs de la psychologie et de l'épistémologie mais aussi à ceux de l'éthique et de la pédagogie. Le TGS apparaît chez Morin comme une métathéorie, c'est-à-dire, comme une théorie de la pensée dont l'objectif est, sur la base d'un concept pluraliste de système, de formuler des règles de valeur générales applicables à tout système et à tout niveau de réalité y comprise ontologique, gnoséologique, éthique, pédagogique qui guident le design éducatif. En même temps les systèmes intégrant la réalité de la pensée et les activités éducatives correspondent à des systèmes spécifiques, caractérisés par leur complexité et leur caractère unique. L'approche complexe de Morin apporte une contribution au débat entre réductionnisme et perspectivisme, abordant des systèmes complexes et globaux, recherchant de façon analytique des aspects essentiels par rapport à sa composition et à sa dynamique, susceptibles d'être généralisés.

16 Ainsi, la théorie de la pensée complexe, que Morin pose comme un nouveau paradigme scientifique où les disciplines sont articulées pour rendre compte d'une réalité qui ne peut être apprivoisée par aucune de ses dimensions prises isolement. La Méthode, compte six volumes, qui abordent différents thèmes articulés pour essayer de décrire ce paradigme complexe. Qu'est-ce que la complexité ? Premièrement, la complexité doit être comprise comme un «tissu » (« complexus »: ce qui est tissé) de constituants hétérogènes associés de façon indissoluble : chez elle se pose le paradoxe de la relation entre l'un et le multiple. En outre, ce tissu comprend des événements, des actions, des interactions, des retours, des déterminations, des risques, qui constituent notre monde phénoménal (Morin, $2005: 21$ ).

17 Concernant le paradigme de la complexité Gómez, Hernández et Ramos affirment que «la complexité chez Morin ne traite pas des problèmes des sciences dites de la complexité, mais avec un nouvel horizon épistémologique pour organiser la pensée ». 
Cette organisation de la pensée permettra d'accéder à la création de savoirs ouvrant la voie à l'émergence d'une nouvelle façon d'être, de penser, d'agir et de vivre ensemble avec la fin de transformer la société. Il s'agit d'un paradigme épistémologique qui défend une stratégie fondamentale, appelée « réforme de la pensée » concevant «la réalité comme un système en changement permanent, en développement et en urgence; reconnaissant le tout comme la somme de ses parties et la spécificité des parties par rapport au tout » (Gómez, Hernández et Ramos, 2016 : 473-474). En accord, on considère que la complexité interprète et relie le monde comme un tissu constitué de multiples unions qui sont liées entre elles pour former des relations systématisées. D'ailleurs, la pensée complexe peut être comprise comme ce qui permet l'intégration de l'humain, en le considérant comme un élément constitutif et constituant de la complexité (Estrada García, 2020 : 5).

Conformément aux lois trouvées dans d'autres disciplines et par l'isomorphisme, Morin élève la compréhension de la réalité éducative, en tant que domaine complexe, réalisant ainsi sa transdisciplinarité et sa multidisciplinarité qui lui appartiennent par essence. Ce primat accordé aux valeurs systémiques peut être mis aussi en relation avec le pragmatisme. "Pragmatisme " vient du grec pragma (le résultat de la praxis, l'action en grec) ce qui atteste du souci d'être proche du concret, du particulier, de l'action et opposé aux idées considérées comme abstraites de l'intellectualisme. Il s'agit d'une pensée radicalement empiriste. Ce qui implique que la notion d'effet pratique est étroitement liée à la question de savoir quels sont les effets d'une théorie pour l'expérience, ce qui, d'ailleurs, est décisif dans le domaine éducatif. Chez Dewey, cela consiste à dire que l'expérience suppose une méthode capable de localiser et d'interpréter les problèmes plus importants dans la vie humaine, ainsi qu'une méthode capable de projeter des solutions pour les confronter et, en conséquence, une méthode de diagnostique moral et politique (Dewey, $1910: 17$ ).

Chez John Dewey et Nel Noddings, les applications les plus célèbres de la méthode pragmatiste en éducation, la méthode pragmatique est présentée comme l'opposé de la théorie spectatoriale de l'éducation. Éduquer n'est pas transmettre de connaissances, mais apprendre à "agir» en connexion avec un projet de vie. En opposition à l'enseignement traditionaliste, Dewey propose une éducation basée sur les propres expériences de l'enfant. Avec cela, Dewey réévalue l'importance de s'occuper des besoins, des préoccupations et des intérêts de l'enfant, ainsi que de la relation avec le monde extérieur, en tant qu'éléments fondamentaux pour fournir une véritable éducation et promouvoir un développement authentique de l'élève. Dans son ouvrage Democracy and Education (1916), Dewey a développé sa théorie de l'école en tant que petite communauté avec des expériences de première main qui permettraient aux enfants de mener des pratiques en coopération et, par conséquent, d'apprendre les attitudes et les compétences d'un bon citoyen. Chez Noddings cela concerne le problème du bonheur. L'éducation, suggérée dans Happines and Education (2005), doit promouvoir la recherche personnelle du bonheur en s'apparentant à une philosophie de vie, voire à une pratique de recherche personnelle envisageant faire du monde un foyer.

Chez Morin dans Enseigner à vivre (2014), le projet d'éducation complexe s'apparente au pragmatisme dans la mesure où est impliquée une recherche à la fois épistémologique et éthique qui cherche à harmoniser le projet éducatif avec la vie. On peut affirmer que la démarche de Morin s'insère en quelque sorte dans le mouvement pragmatiste en ce 
qu'il intègre en grande partie les préoccupations : approche critique des dualismes (par exemple), proximité avec une orientation perspectiviste, souci de partir d'expériences isolées, refus de la spéculation abstraite et volonté d'articuler en permanence réflexion politique pour l'exercice d'une citoyenneté démocratique et l'éducation. Il faut cependant préciser que l'approche du pragmatisme avec laquelle Morin a le plus d'affinités est celle de Noddings. Morin partage avec Noddings une critique des savoirs compartimentés et un projet de démocratie globale qui s'enracine dans la mise en relief d'une pensée complexe dans un cas et une pensée pluraliste dans l'autre afin de mieux tenir compte de la relation d'interdépendance. On retrouve chez Morin, comme chez Noddings, un même souci pour la problématique du développement humain et corrélativement pour la question éducative.

\section{Points de rencontre entre l'éducation complexe et l'éthique du care}

21 Quand on veut associer l'idée de "complexité » au problème éducatif, une première possibilité est de mettre l'accent sur la pensée. Dans ce cas-là, la complexité implique une redéfinition de l'approche pédagogique basée sur la fragmentation de la connaissance à travers différentes matières. Selon Morin, la connaissance, telle qu'elle est transmise et enseignée aujourd'hui, perçoit mal la complexité : soit elle sépare les données, soit elle n'y voit que confusion. Pour Morin elle est toujours une traduction et une reconstruction qui comportent le risque de l'erreur. Elle est gouvernée en profondeur par ce qu'on peut appeler le paradigme de disjonction, autrement dit : nous voulons comprendre un tout complexe à partir de ses éléments constitutifs, séparés de leur environnement et des ensembles dont ils font partie. La pensée analytique isole les questions et évite les contradictions. D'après le point de vue de la pensée analytique, par exemple, les concepts d'autonomie et de dépendance guidant l'éducation actuelle sont conçus en tant que termes contradictoires.

Lorsque Morin s'est mis en devoir de penser l'éducation du futur, une éducation dans laquelle la tâche la plus importante est celle d'articuler, d'organiser et de percevoir et concevoir le contexte, le global (la relation tout/parties), le multidimensionnel, le complexe (Morin, 2008), nous prenons conscience du fait qu'il n'existe pas des principes directeurs éthiques qui permettent de recréer le lien entre les connaissances sans lequel elles restent vides de sens. Dans une telle situation, pour Morin la réforme de l'éducation doit conduire à une réforme de la pensée pour la bonne compréhension entre les peuples, les nations, les religions, nécessaire au bien vivre. Cette réforme de la pensée se base pour Morin sur la nécessité d'admettre le caractère transdisciplinaire de la connaissance et le besoin de relier l'objet au sujet et à son environnement. À travers la collection de textes qui comprennent La Méthode, Morin tente de concevoir comment lier ce qui était considéré dans le paradigme éducatif jusqu'ici comme disjoint, à travers une "approche dialogique" qui permet de connaître à la fois les globalités et le particulier. Étendue à l'éducation, la pensé complexe suppose «de réformer la pensée pour réformer l'enseignement et de réformer l'enseignement pour réformer la pensée ", ainsi qu'il le préconise dans La tête bien faite (1999).

La crise de la civilisation à laquelle fait référence Morin donne aussi une visibilité publique au déficit et aux difficultés de l'éducation. Aussi bien la formulation de la pensée complexe que l'éthique du care seraient tous les deux l'expression d'une crise de 
la responsabilité humaine qui demanderaient des solidarités en même temps qui expliqueraient l'émergence de la pensée complexe et du care en tant que revendications d'une autre façon de concevoir la relation en éducation (aussi bien d'un point de vue éthique qu'épistémologique). D'où pour Morin la nécessité d'établir des communications et des liens entre les deux branches séparées de la culture: l'humanisme et la science. La principale conséquence, d'un point de vue éthique, c'est que la pensée complexe nous incite à "l'éthique de la compréhension ».

En cela, un auteur comme Morin insiste sur la façon dont l'éducation complexe envisage toute activité comme inscrite dans une tension, comme des opérations effectuées dans le cadre des situations problématiques. Ainsi, l'action éducative ne répond pas au schéma de poursuivre des fins données ou d'appliquer des normes et la complexité apparaît comme une ouverture aux façons d'agir. C'est dans ce cadre que prennent sens le souci de mettre l'accent sur la solidarité et la responsabilité comme vecteurs de reconstruction de l'éducation face à la fragmentation des situations problématiques et l'insistance sur la complexité de la pensée que Morin propose d'appréhender en termes de problématisation. La pensée est ainsi saisie comme processus d'interaction entre l'être humain et son environnement, en vue d'obtenir des informations permettant de vivre.

25 Il est important de préciser que la philosophie du care n'est pas homogène et qu'ils existent différentes définitions du care ainsi que sur son statut épistémologique. Entre toutes les définitions possibles du care, nous allons travailler sur celle de Tronto et Fisher (1990) : « une activité caractéristique de l'espèce humaine qui inclut tout ce que nous faisons en vue de maintenir, de continuer ou de réparer notre "monde » de telle sorte que nous puissions y vivre aussi bien que possible. Un monde qui inclut nos corps, nos individualités et notre environnement, et que nous cherchons à tisser ensemble dans un maillage complexe qui soutient la vie » (traduction de Moliner et al., 2009).

Le but de la réforme de l'éducation, qui est finalement le «bien vivre » de chacun et de tous, les enseignants et les enseignés, nécessite de part et d'autre une régénération de l'Eros. Cet Eros chez Morin fait appel à une action éthique. Pour lui l'éducation doit être basée sur une «anthropo-éthique ». En effet, on ne peut pas atteindre l'autonomie qui nous fait humain avant d'apprendre une langue, une culture, un savoir. Notre autonomie se nourrit donc de la dépendance: d'une éducation, d'une société, d'une culture. Nous dépendons d'un cerveau et de notre héritage génétique. Cependant, nous avons la capacité de prendre des distances et d'exercer notre capacité de prise de décision, au-delà du conditionnement social, culturel et génétique. L'autonomie et la dépendance doivent alors être visualisées dans un processus de compromis et d'antagonismes entre instances opposées, mais qui pourtant se génèrent dans un ensemble de tensions (Morin, 1994).

\section{L'apport de l'éducation complexe à l'établissement d'une communauté éducative}

«Participer» n'est pas seulement prendre part aux activités d'un groupe; la participation implique aussi de se sentir faire partie des activités, assumer une action et avoir profit des actions. Autrement dit, la « participation » conseillée par l'Unesco pour promouvoir l'inclusion n'implique pas seulement que les élèves deviennent sujets actifs de leur apprentissage, mais aussi qu'ils profitent de leur éducation, assument leur 
responsabilité dans les processus d'enseignement-apprentissage et se sentent comme des membres à part entière de la communauté éducative. L'impact de la réforme de l'éducation peut être encore plus puissant et peut réveiller les aspirations et le sens de la responsabilité innée en chacun des agents éducatifs.

À la différence de la pensée analytique, la stratégie épistémique du paradigme de la complexité de Morin renvoie au cadre théorique de «l'éco-organisation» : cela signifie non pas de focaliser des faits statiques et isolés, mais des processus dynamiques d'interaction. Sous ce prisme là le concept d'autonomie est conçu d'une façon complexe, c'est à dire, comme dépendant des conditions culturelles, sociales et de l'environnement. Ce sont donc des systèmes ouverts : tout système, pour rester en vie doit échanger avec son environnement ; sinon, il est étouffé et détruit.

Il y a donc un principe d'organisation (éco-organisation) à caractère complexe, dans lequel le tout est dans la partie qui est dans le tout, et dans lequel chaque partie pourrait alors pouvoir régénérer le tout. Ainsi, on doit focaliser, par exemple, chaque objet ou instrument non pas comme une chose isolée, mais comme un réseau. De cette façon, chaque instrument articule l'ensemble du système qui constitue la pensée, et son utilisation nous implique avec la totalité. D'un autre côté, ce système n'est pas une entité qui fonctionne dans le vide. Elle implique une culture, des valeurs, des directives et des codes, des hypothèses sous-jacentes, que nous acceptons normalement sans critique réfléchie. C'est pourquoi chaque transfert épistémologique implique un transfert éthique en même temps. Nous pouvons synthétiser cette proposition épistémique de la manière suivante: pour la pensée analytique, réductrice, simplificatrice, tout est ce qu'il est. Néanmoins, pour la pensée complexe de Morin, rien n'est juste ce qu'il est : tout est plus que ce qu'il est, car il existe un réseau qui implique son contexte.

L'éthique ne nous dit pas quoi faire dans chaque situation, mais nous oblige plutôt à appliquer l'intelligence pour les évaluer. Morin nous prévient qu'il ne s'agit pas d'éliminer les antagonismes ou de parvenir à une harmonie permanente, mais de reconnaître le conflit comme partie intégrante de la complexité. Le principe de l'écologie de l'action est un pari pour assumer la responsabilité de l'action humaine dans sa dimension éthique et politique. Certes, dans le contexte éducatif actuel, il n'est pas approprié de comprendre que les individus sont ceux qui devraient assumer la responsabilité des conséquences imprévisibles et des sous-conséquences de leur action (les élèves leur échec académique, les enseignants, l'échec de leurs élèves, les chefs d'établissement, l'échec des enseignants). Au contraire, le défi proposé par Morin est d'assumer la «responsabilité conjointe de coopération conjointe».

L'action éducative est décision et choix, et dans la notion de décision il y a la conscience du risque et de l'incertitude qui peut porter atteinte aux enfants les plus vulnérables. Dans ce point Morin fait intervenir le concept de «l'écologie de l'action »: dès qu'un individu entreprend une action, quelle qu'elle soit, celle-ci commence à échapper à ses intentions. Cette action entre dans un univers d'interactions et c'est finalement l'environnement qui s'en saisit dans un sens qui peut devenir contraire à l'intention initiale. Cela nous oblige à contrôler l'action, à essayer de la corriger. En somme, l'écologie de l'action signifie "tenir compte de la complexité qu'elle comporte, avec aléas, hasards, initiatives, décisions, imprévus, et elle nécessite la conscience des dérives et des transformations » (Morin, $2000: 104$ ). 
32 Le principe de "l'écologie de l'action"soulevé par Edgar Morin dans son éthique complexe, est un pari pour prendre en charge de manière responsable l'action humaine ; en ce sens que la proposition morinienne est un engagement transformateur pour la connaissance et l'action éducative dans une dimension éthique et politique. "L'écologie de l'action », principe inventé par Edgar Morin (2004), implique que toute action échappe à la volonté de son auteur en entrant dans le jeu des inter-rétro-actions du médium en qui intervient, rend compte d'une question cruciale qui implique la relation entre "les motivations d'une action» et «ses conséquences». Une action éthique ainsi comprise, va au-delà d'un sujet tourné sur ses propres convictions, d'une action individuelle sans relations, sans contexte. Au contraire, la barbarie, l'incertitude, nous pousse à réfléchir à la responsabilité éthique de l'action; et cela nécessite une complexité dans la manière de penser. Le monde d'aujourd'hui a besoin de connaître quelle rationalité, quelle relationnalité; ou plutôt, parier sur la rationalité ouverte, plurielle, dialogique qui rend possible la rationalité / complémentarité relationnelle.

Cette éthique dont nous parle Morin a comme sources la solidarité et la responsabilité. Elle doit se former dans les esprits à partir de la conscience que l'humain est à la fois individu, partie d'une société et partie d'une espèce. Elle doit comporter le développement conjoint des autonomies individuelles, des solidarités communautaires et de la conscience d'appartenir à l'espèce humaine. Dans ce sens «l'éthique individu/ société nécessite un contrôle mutuel de la société par l'individu et de l'individu par la société, c'est-à-dire la démocratie ». À partir de cela s'esquissent les deux grandes finalités éthico-politiques du nouveau millénaire: «établir une relation de contrôle mutuel entre la société et les individus par la démocratie, accomplir l'humanité comme communauté planétaire ». L'enseignement doit contribuer, non seulement à une prise de consciente de notre terre patrie, mais aussi permettre que cette conscience se traduise en une « volonté de réaliser la citoyenneté terrienne » (Morin, $2014: 107$ ).

La préoccupation morale qui anime centralement les éthiques du care est aussi celle de la responsabilité. Pour elles, "prendre soin signifie en effet d'abord répondre de manière appropriée à ce à quoi et à ceux à qui nous sommes liés. Mais, dans la mesure où le sujet de cette responsabilité comme le contexte dans lequel il agit et les relations qui sont les siennes sont toujours particulières, il n'est guère possible de définir de manière générale en quoi consiste une réponse moralement appropriée " (Garrau, 2014 : 46). Dans la perspective du care c'est en effet de la perception fine du contexte que dépend l'action appropriée. Cela signifie qu'en matière de morale, la bonne réponse est toujours particulière et ne peut être définie a priori. L'action morale est irréductible à l'application de règles et dépend fondamentalement de la situation, elle n'a ni la simplicité ni la clarté que lui prête l'éthique de la justice. Concevoir l'action morale comme l'application d'une règle générale et abstraite préalablement donnée risque au contraire d'empêcher l'agent de percevoir la particularité et la complexité du contexte (Garrau, 2014 : 47).

En distinguant différents aspects du processus comme le propose Tronto, la perspective du care se dote de capacités descriptives pour dire comment le monde éducatif peut tenir en s'appuyant sur un travail méconnu. Les activités du care font «tenir » un monde commun sans nier sa complexité, car elles contiennent et projettent sur ce monde une conception de ce qui fait ce commun, et qui n'est généralement pas compris dans les descriptions courantes du monde dit commun, encore moins ses définitions officielles. (Molinier et al., 2009: 101). Également, la pensée complexe permet de 
percevoir, concevoir et penser d'une façon organisée tout ce qui nous entoure (Morin 1981 : 105), les principes dialogiques et de récursivité qui guident, entre autres, la pensée complexe et qui peuvent être mis en relation avec les phases du care.

Fisher et Tronto ont décrit quatre phases du care auxquelles il faut ajouter des dimensions morales : le fait de se soucier de quelqu'un ou quelque chose (caring about); prendre soin de quelqu'un (caring for); de soigner quelqu'un (care giving); d'être l'objet du soin (care receinving); qui se correspondent avec les principes dialogique et de récursivité préconisés par Morin. Le fait de se soucier de quelqu'un implique en premier lieu la reconnaissance d'un besoin de care (principe dialogique); est nécessaire la qualité morale spécifique de l'attention aux besoins de l'autre. Prendre soin suppose d'assumer la responsabilité du travail de care qu'il faut accomplir ; son corollaire moral est la responsabilité. Le fait de soigner est le travail concret du care auquel il s'attache la qualité morale de la compétence professionnelle. Finalement être objet du soin est la réponse de la personne qui a bénéficié du care. La qualité morale ici est la capacité de réponse. Cette proposition regroupe plusieurs disciplines et perspectives: une catégorie psychologique (le souci de soi); une catégorie sociologique (le souci des autres); et des catégories économiques et politiques (le souci du monde).

Quand nous définissons l'éducation ainsi, notre conception des relations éducatives change. Tout d'un coup, on ne voit plus simplement le monde éducatif comme un ensemble d'individus autonomes poursuivant des fins rationnelles et des projets de vie, mais on voit le monde comme un ensemble de personnes prises dans des réseaux de care et engagées à répondre aux besoins de care qui les entourent (Molinier et al., 2009 : 39). Le care concerne toutes ces attitudes, mais aussi la capacité à prendre des responsabilités. Les activités de care doivent permettre l'expression des points de vue de tous les acteurs impliqués. Un bon care ne pourra être défini sans que soient pris en compte les points de vue des différents acteurs du care sur leurs pratiques et les contextes dans lesquels celles-ci sont effectuées (Garrau, 2014: 29). Pour Morin la conflictualité résultant de la relation et des différentes perspectives des individus ne peut être totalement abolie, mais elle peut être minorée ou surmontée par la compréhension. La perspective de la complexité met alors en garde contre les processus "d'auto-éco-organisation", c'est-à-dire, contre les processus que ne tient pas en compte le contexte et autrui. Il s'agit, tout d'abord, de concevoir les processus éducatifs dans une perspective dialogique (Morin, 1986) tenant en compte instances à la fois antagonistes et complémentaires.

\section{L'apport de l'éducation complexe au projet d'une éducation inclusive}

Cette réflexion a permis d'explorer deux lignes possibles, l'éthique et l'épistémologique, par lesquelles nous pouvons aborder la complexité de l'éducation. On a montré comme Morin cherche à étendre l'idée purement didactique de l'éducation vers une conception plus complète et plus complexe de cette réalité dont la vocation est d'éviter une simplification excessive des processus d'enseignement-apprentissage.

En mettant en relation la pensée complexe de Morin avec la philosophie pragmatiste et du care, nous déduisons que l'identification réductrice de l'éducation avec pour devoir d'accomplir les objectifs d'un programme, produit des aliénations et laisse bon nombre 
d'élèves en dehors du système. Cette conception de l'éducation ne permet pas de tenir compte des relations qui composent la réalité éducative et où la vulnérabilité est en jeu. Or, l'éducation complexe met l'accent sur l'importance de la relation. Face aux limites de cette conception de l'éducation, l'éducation complexe est guidée par une éthique de la responsabilité et mise sur l'application d'une "écologie de l'action » en tant que principe de respect et de reconnaissance des contextes, sachant que l'application est toujours conditionnée par les conséquences et les situations de réalités concrètes. Elle propose des transformations dans les sphères éducative, politique et sociale qui favorisent une nouvelle compréhension globale et, en conséquence, l'inclusion en éducation.

Au-delà du paradigme de l'éducation basé sur la transmission de savoirs compartimentés, l'instruction et l'accomplissement d'objectifs abstraits, l'action éducative doit être associée à son environnement, affronter la contradiction, respecter la multi-dimensionnalité des êtres et des choses, travailler et dialoguer avec l'incertitude. L'éducation implique de la complexité, elle ne nie pas les différences ni les difficultés. Sous ce prisme le problème de l'éducation n'est pas d'accomplir un programme et les principes directeurs ne sont pas basés sur une éthique du devoir, mais plutôt d'une éthique de la responsabilité ou du care. Elle va au-delà des raisons individuelles et mise sur une rationalité fondée sur un tissu pluriel. En d'autres termes, la responsabilité de savoir si les conséquences de nos actions sont en adéquation avec ce que nous aimerions pour nous-mêmes, pour la société, pour la planète. L'écologie de l'action s'exprime au moyen des relations, interactions et commentaires générés par le contexte. Ce principe constitue le fond éthique d'une éducation démocratique et pluraliste permettant de penser l'inclusion non pas comme une solution aux défauts des systèmes éducatifs, mais comme l'essence de l'éducation.

Cette voie d'une éducation complexe permet de reconceptualiser le terme «responsabilité » dans un cadre déterminé par le contexte et de conséquences. Cela implique aussi un pari pour la fraternité, qui n'est rien d'autre qu'une coopération solidaire entre l'individu / la société / l'espèce. Cela nécessite, d'une part, une réforme de la pensée, et d'autre part une institutionnalisation politique et une institutionnalisation dans la praxis collective comprise comme tâche de coopération solidaire où tous les agents éducatifs sont nécessairement impliqués.

\section{BIBLIOGRAPHIE}

Berntalanfy, L. (2012). Théorie générale des systèmes. Paris : Dunod.

Dewey, J. (1910). The influence of Darwin on Philosophie. And other essays in contemporary thought. New York : Herny Holt and company.

Estrada García, A. (2020). Los principios de la complejidad y su aporte al proceso de enseñanza, Ensaio : aval. Pol. Publ. Educ. Rio de Janeiro, 1-20.

Fabre M. (2009). Philosophie et pédagogie du problème. Paris : Vrin. 
García, A. y Jover, G. (2019). Felicidad, educación y democracia en el pensamiento de Nel Noddings. Encounters in Theory and History of Education, vol.20, ํํ1, 24-44.

Garrau, M. (2014). Care et attention. Paris : Puf.

Gomez, C. ; Hernández, M. ; Ramos, R. (2016). Principios epistemológicos para la enseñanza aprendizaje, según el pensamiento complejo de Edgar Morin. Pueblo Continente, 471-479.

Grandjean, N., Loute, A. (2019). Valeurs de l'attention: Perspectives éthiques, politiques et épistémologiques. Namur : Presses universitaires du Septentrion.

Haddock Seigfried, C. (1996). Pragmatism and Feminism. Reweaving the social fabric. ChicagoLondon: The University of Chicago Press.

Kant, E. (1986). « Propos de pédagogie ». Euvres philosophiques III : les derniers écrits. Paris : Gallimard.

Le Goff, A. (2013). Care et démocratie radicale. Paris : Puf.

Mbembe, A. (2006). Necropolitique, Traversées, diasporas, modernités. Raisons politiques, $\mathrm{n} 221$, 29-60.

Molinario, J. (2017). Surmonter les dualismes par l'éducation intégrale. Transversalités, ㄲำ141, 2, 21-33.

Molinier, P.; Laugier, S.; Paperman, P. (2009). Qu'est-ce que le care? Souci des autres, sensibilité, responsabilité. Paris: Payot.

Morin E. (1981). La Méthode I. La Nature de la nature. Paris : éd. du Seuil.

Morin E. (1986). La Méthode III. La Connaissance de la connaissance. Paris : éd. du Seuil.

Morin E. (1991). La Méthode IV. Les Idées. Leur habitat, leur vie, leur mœurs, leur organisation. Paris : éd. du Seuil.

Morin E. (2004). La Méthode VI Éthique. Paris : éd. du Seuil.

Morin, E. (1994). La Complexité humaine. Paris : Flammarion.

Morin E. (1999). La tête bien faite. Repenser la réforme, réformer la pensée. Paris : éd. du Seuil.

Morin, E. (2000). Les sept savoirs nécessaires à l'éducation du futur. Paris : Éditions du Seuil.

Morin, E. (2014). Enseigner à vivre. Paris: Actes Sud-Playbac.

Noddings, N. (2005). Educating Citizens for Global Awareness. Teachers College Press.

Noddings, N. (2005). Happiness and Education. Cambridge : Cambridge University Press.

Noddings, N. (2013). Education and democracy in the 21st century. New York, NY: Teachers College Press.

Tronto J. (2009). Un monde vulnérable. Pour une politique du care. Paris : La Découverte.

Tronto, J.C, Fisher, B. (1990). Toward a Feminist theory of Caring. E. Abel \& M. Nelson (Eds.) Circles of Care. Albany, NY : Suny Press, 36-54.

UNESCO (2009). Principes directeurs pour l'inclusion: assurer l'accès à l'Éducation Pour Tous. Paris: UNESCO. 


\section{NOTES}

1. Comme, par exemple, c'est le cas de Hannah Arendt dans « La crise de l'éducation ", extrait de La crise de la culture. Paris : Gallimard, 2007.

2. Cette idée, inspirée par l'ouvrage d'Achile Mbembe Necropolitique (2005), permet de définir l'éducation comme l'éducation qui est contraire à sa finalité essentielle : celle d'enseigner à vivre. Dans ce sens-là, la necro-education, conduit aussi à la chosification de l'être humain à travers des forces économiques et idéologiques qui mercantilisent et réifient la connaissance.

\section{RÉSUMÉS}

La convergence entre la pensée pragmatiste et les approches du care a été peu abordée dans le contexte francophone. C'est au prisme de la pensée complexe d'Edgar Morin que ce texte met en lumière les points de rencontre entre pragmatisme et philosophie du care. Morin élabore une épistémologie éducative dont les approches du care peuvent se réclamer. Cet article explore la façon dont cette épistémologie met en évidence la relation en éducation, conçue comme une organisation "écologique» entre défense des valeurs du care et promotion du continuum théorie-pratique pédagogique. Il s'agit par-là de mettre en lumière les raisons pour lesquelles la complexité du projet de Morin et son apport à la réflexion pédagogique sur le care sont pensés. Mais il s'agit aussi de signaler la contribution de ce projet à la promotion de politiques et pratiques éducatives inclusives.

The convergence between pragmatist thinking and care approaches has been little discussed in the French-speaking context. It is through the prism of Edgar Morin's complex thinking that this text highlights common ground between pragmatism and care philosophy. Morin develops an educational epistemology in which care approaches can be claimed. This article explores how this epistemology highlights the relationship in education, conceived as an "ecological" organization between the defense of care values and the promotion of continous between theory and practice. The aim of this is to highlight the reasons why Morin's project complexity and its contribution to educational reflection about care are considered. It is also pointing out the contribution of this project to the promotion of inclusive educational policies and practices.

\section{INDEX}

Mots-clés : éducation complexe, épistémologie, éthique du care, relation éducative

Keywords : complex thinking, eco-organization, ethics of care, educational relationship

\section{AUTEUR}

\section{ALICIA GARCIA}

Universidad Complutense de Madrid, Groupe de Recherche Cultura Cívica y Políticas Educativas 